\title{
An Optimal Fuzzy Logic Controller tuned with Artificial Immune System
}

\author{
S. N.Omkar ${ }^{1 \mathrm{a}}$, Nikhil Ramaswamy ${ }^{1 \mathrm{~b}}$, R.Ananda ${ }^{2}$,Venkatesh N.G. ${ }^{3}$ and J.Senthilnath ${ }^{1}$ \\ ${ }^{1}$ Department of Aerospace Engineering, Indian Institute of Sciene, Bangalore-560012 \\ ${ }^{2}$ Department of Aerospace Engineering, Indian Institute of Technology, Kharagpur-721302 \\ ${ }^{3}$ Department of Electronics and Communication Engineering, National Institute of \\ Technology Karnataka Surathkal, Mangalore -575025 \\ $\left\{\right.$ \{amkar@aero.iisc.ernet.in,$\underline{{ }^{b} \text { nrghatta@gmail.com\} }}$
}

\begin{abstract}
In this paper, a method for the tuning the membership functions of a Mamdani type Fuzzy Logic Controller (FLC) using the Clonal Selection Algorithm(CSA) a model of the Artificial Immune System(AIS) paradigm is examined. FLC's are designed for two problems, firstly the linear cart centering problem and secondly the highly nonlinear inverted pendulum problem. The FLC tuned by AIS is compared with FLC tuned by GA. In order to check the robustness of the designed FLC's white noise was added to the system, further, the masses of the cart and the length and mass of the pendulum are changed. The FLC's were also tested in the presence of faulty rules. Finally, Kruskal Wallis test was performed to compare the performance of the GA and AIS. An insight into the algorithms are also given by studying the effect of the important parameters of GA and AIS.
\end{abstract}

Keywords: Fuzzy logic Controller,Artificial Immune System, Genetic Algorithms

\section{Introduction}

Fuzzy Logic Controllers (FLC) have gained prominence in recent years because of its ability to control devices which tend to imitate human decision making. This allows for a control design which is simple and accurate. A FLC is also efficient as it captures the approximate and qualitative boundary conditions of a system's variables by fuzzy sets with a membership function. The FLC controls a system by a set of linguistic IF-THEN rules and has been shown to be robust and straightforward to implement $[1,2]$. Due to these advantages FLC's have been successfully applied to various industrial applications [3-6].

However, the main challenge of designing the FLC lies in choosing optimal fuzzy parameters for its membership functions (MF). Previously, the task of generation of MF's were done by trial and error techniques, unfortunately this is a te- 
dious approach. Since this task is a natural candidate for stochastic approaches like Genetic Algorithms (GA). Various researchers have extensively used GA's for tuning the MF's of a FLC[7-9]. However, despite their advantages of evolving optimal solutions for wide variety of problems, as reported in [10], GA has the disadvantage of getting stuck in the local minima and may take large time to obtain a near optimal solution. This has motivated researchers to study other evolutionary algorithms to overcome the disadvantages of GA.

In this paper a relatively new evolutionary computation paradigm-Artificial immune system (AIS)[11] is used to tune the Mamdani type fuzzy controller's anecedent and consequent parameters. In this work the fuzzy inference system(i.e Mamdani) tuned by AIS, is applied to a FLC to show the efficacy of the proposed method. In our study we use the Clonal selection algorithm (CSA) which is one of the most extensively used AIS model [12]. This method of CSA has been successfully used for solving various real world and optimization problems [13]. To show the efficacy of AIS in tuning of the FLC, two problems are considered. The first is the cart centering problem (CCP), in which the objective of the controller is to bring a cart with initial displacement and velocity to rest [14]. The second problem is the classic benchmark problem in control i.e the inverted pendulum (IP) problem [15] in which the objective is to bring a pendulum on a cart to rest from an initial angular displacement and angular velocity. On designing the FLC's using GA and AIS we compare the performance of the FLC tuned by the two algorithms based on statistical comparison, robustness, stability and the ability of the FLC to work in the presence of faulty fuzzy rules. Further, a performance comparison between the two algorithms is presented and an insight into determining the optimal parameters for each algorithm is analyzed.

\section{Fuzzy Logic Controller}

FLC is based on the concept of fuzziness in which, rather than allowing a system to have a value of 0 or 1 , fuzzy allows the system to have degrees of membership functions over the range $[0,1]$. The basis for design of a FLC is the linguistic IF-THEN rules for eg: $I F I_{1}$ is $B_{i 1} A N D I_{2}$ is $B_{i 2}$ THEN $O$ is $D_{i}$, where $I_{1}, I_{2}$ are the inputs and $O$ is the output of the FLC. $B_{i 1}, B_{i 2}$ and $D_{i}, i=\{1, \ldots, \mathrm{n}\}$ are linguistic values presented in the fuzzy subsets of the universe of discourse.

In this paper, for the CCP, the values of $\mathrm{x}($ displacement $)(\mathrm{m})$ and $\mathrm{v}($ velocity $)(\mathrm{m} / \mathrm{s})$ are scaled to the interval of $[-5,5]$ and $\mathrm{F}($ Force $)(\mathrm{N})$ from $[-75,75]$. For the IP, the values of $\theta$ (angular displacement)(rad) and $\dot{\theta}$ (angular velocity) $(\mathrm{rad} / \mathrm{s})$ are scaled to the interval of $[-0.5,0.5]$ and $\mathrm{F}$ from $[-5,5]$. The inputs and outputs of the FLC are composed of seven linguistic terms NB(Negative Big), NM(Negative Medium), NS(Negative Small), Z(Zero), PS(Positive Small), PM(Positive Medium) and PB(Positive Big). This set of linguistic terms forms a fuzzy partition of input and output spaces. In our study we use the Gaussian symmetrical membership functions(GMF).The fuzzy IF-THEN rules for the two 
problems examined here i.e. CCP and IP are the same as shown in Table1. The defuzzification method used is the centroid of area.

Table 1. Rule Base

\begin{tabular}{llllllll}
\hline$I_{I}$ & NB & NM & NS & Z & PS & PM & PB \\
\hline$I_{2}$ & & & & & & & \\
\hline PB & Z & NS & NM & NB & NB & NB & NB \\
PM & PS & Z & NS & NM & NM & NM & NB \\
PS & PM & PS & Z & NS & NM & NM & NB \\
Z & PB & PM & PS & Z & NS & NM & NB \\
NS & PB & PM & PM & PS & Z & NS & NM \\
NB & PB & PB & PB & PM & PS & Z & NS \\
NM & PB & PB & PM & PB & PS & PM & Z \\
\hline \hline
\end{tabular}

\section{Problem Formulation}

In this section we discuss the two problems for which FLC's are designed.

\subsection{Cart Centering Problem}

In this problem a cart of mass $\mathrm{M}$ moves on a one dimensional track. The input variables for this problem are the cart's location on the track, $\mathrm{x}$, and the cart's velocity, $\mathrm{v}$. The objective of the controller is to apply a suitable force $\mathrm{F}$ which will bring the cart to rest, i.e $\mathrm{x}=0$ and $\mathrm{v}=0$ from an initial displacement and velocity. The equations of motion for the cart are

$$
\begin{aligned}
& x(t+\tau)=x(t)+\tau v(t) \\
& v(t+\tau)=v(t)+\tau F(t) / M
\end{aligned}
$$

where $\tau$ is the time step. In this paper $\tau$ is $0.02 \mathrm{~s}$ and $\mathrm{M}$ is $1 \mathrm{~kg}$ and the initial conditions for $\mathrm{x}$ and $\mathrm{v}$ is $1 \mathrm{~m}$ and $1 \mathrm{~m} / \mathrm{s}$ respectively.

\subsection{Inverted Pendulum Problem}

The IP is a classic benchmark problem used in control literature due to its high nonlinear dynamics. Moreover this system has fewer control inputs than the degrees of freedom, making its control challenging. The IP is also a representative of a class of altitude control problems whose goal is to maintain the desired vertically oriented position at all times [2].

The input variables for this problem are the angular displacement of the pole, $\theta$ and the angular velocity, $\dot{\theta}$. The objective of the controller is to apply a suitable 
force $\mathrm{F}$ on the cart which will bring the pendulum to rest at vertical position i.e $\theta=$ 0 and, $\dot{\theta}=0$ from an initial $\theta$ and , $\dot{\theta}$. Here we are concerned with the control of the angular position of the pendulum and not the position or velocity of the cart. The cart travels in one direction along a frictionless track . The equations describing the angle dynamics of the IP are:

$\dot{\theta}=\frac{d \theta}{d t}$

$\ddot{\theta}=\frac{g \sin (\theta)-\left(\cos (\theta) / m_{p}+m_{c}\right)\left(m_{p} l \dot{\theta}^{2} \sin (\theta)+F(t)\right.}{\frac{4 l}{3}-\left(m_{p} l(\cos (\theta))^{2} / m_{p}+m_{c}\right)}$

where $g$ is $9.8 \mathrm{~m} / \mathrm{s} 2$, mass of pendulum $\left(\mathrm{m}_{\mathrm{p}}\right)$ is $0.1 \mathrm{~kg}$, mass of $\operatorname{cart}\left(\mathrm{m}_{\mathrm{c}}\right)$ is 0.9 $\mathrm{kg}$ and length of pendulum is $0.5 \mathrm{~m}$. the initial condition for $\theta$ and, $\dot{\theta}$ are $0.1 \mathrm{rad}$ and $0.1 \mathrm{rad} / \mathrm{s}$ respectively.

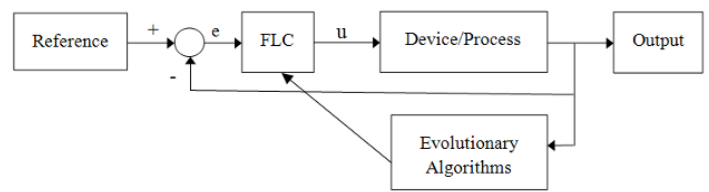

Fig.1. Block structure of the FLC optimizing process using evolutionary algorithms

Fig. 1. shows the block structure of the FLC optimizing process using evolutionary algorithms here, GA or AIS. 'e' represent the error between the output and reference and ' $u$ ' represents the control input. Device/Process refers to either CCP or IP. Since there are $21 \mathrm{MF}$ 's and 49 rules, there are a total of 42 decision variables to be optimized. In the next two sections we discuss the applicability of GA and AIS to tune the FLC's.

\section{Genetic Algorithm}

The GA are based on the Darwinian principle of survival of fittest and the natural process of evolution through reproduction. In GA the solution to a given problem is encoded in the form of strings called chromosomes. Each chromosome consists of a set of genes that contain a set of values for the optimization variables. GA works by composing a random initial population $P$ consisting of a set of chromosomes each representing a possible solution to the problem. The fitness of each chromosome is then computed by evaluating it against the objective function. The present population then evolves towards better solutions through genetic operators namely reproduction, cross over and mutation. For tuning the FLC using GA, all the antecedent and consequent parameters in the d-dimensional space is searched. The solution representation in GA is as shown below:

$$
\mathrm{P}_{\mathrm{a}}=\left[\sigma_{\mathrm{i} 1}{ }^{\mathrm{k}} \sigma_{\mathrm{i} 2}{ }^{\mathrm{k}} \sigma_{\mathrm{o}}{ }^{\mathrm{k}} \mathrm{c}_{\mathrm{i} 1}{ }^{\mathrm{k}} \mathrm{c}_{\mathrm{i} 2}{ }^{\mathrm{k}} \mathrm{c}_{\mathrm{o}}{ }^{\mathrm{k}}\right] \text {, where } \mathrm{k} \in[1, \ldots 7]
$$


Where $\sigma_{i 1}, \sigma_{i 2}, c_{i 1}$ and $c_{i 2}$ represents the deviation and centre for the two inputs of the GMF's and $\sigma_{\mathrm{o}}$ and $\mathrm{c}_{\mathrm{o}}$ represents the deviation and centre for the outputs of the GMF's. The most important step in applying evolutionary algorithms is to establish a measure of its fitness, to accurately evaluate the performance of the controller. However the task of defining the fitness function is application specific. In this paper we use the Root Mean Square Error(RMSE) given by Eq.(5).

$$
\mathrm{RMSE}=\sqrt{\frac{\sum_{\mathrm{i}}^{\mathrm{N}} \mathrm{e}^{2}}{\mathrm{~N}}}
$$

Where e is the error in trajectory of displacement for CCP and e is error in trajectory of angular displacement for IP and $\mathrm{N}$ is the number of points. The pseudocode for optimization of FLC using GA is as follows:

1. The initial population is initialized with random antecedent and consequent parameters of the FLC in the d-dimensional space

2. The fitness of each chromosome is calculated according to the objective function using $\mathrm{Eq}(5)$

3. The population is arranged in order of their fitness values and the chromosome with best fitness is reproduced.

4. Crossover and mutation operators are carried out on consecutive chromosomes. This is done ensuring that no chromosome is chosen twice.

5. The fitness value of the new population is calculated and it is ensured that only the fittest chromosomes enter the next generation.

6. Steps 2-5 are carried out till maximum number of generations is reached.

\section{Artificial Immune System}

AIS is composed of intelligent methodologies inspired by the natural immune system which are used to solve the real world problems [16]. The clonal selection algorithm is part of AIS based on clonal expansion and affinity maturation [11]. The clonal selection theory describes that when an antigen (Ag) is detected, antibodies $(\mathrm{Ab})$ that best recognize this $\mathrm{Ag}$ will proliferate by cloning. This immune response is specific to each Ag. The immune cells will reproduce in response to a replicating Ag until it is successful in recognizing and fighting against this $\mathrm{Ag}$. Some of the new cloned cells will be differentiated into plasma cells and memory cells. The plasma cells produce $\mathrm{Ab}$ and promotes genetic variation when it undergo hypermutation. The memory cells are responsible for the immunologic response for future Ag invasion. Subsequently, the selection mechanism will keep the cells with the best affinity to the Ag in the next generation.

Based on the clonal selection principle, an algorithm is developed in which various immune system aspects are taken into account such as: maintenance of the memory cells, selection and cloning of the most stimulated cells, death of nonstimulated cells and re-selection of the clones with higher affinity and generation and maintenance of diversity.

Since our problem is in continuous domain space, the antibody chains are binary coded. During simulations, each value in the chain is encoded by 23 bits. The 
fitness of each chain is calculated after it was decoded to real values. The initial population is cloned and the cloned chains undergo the process of hypermutation and re-selection.

Hypermutation is an important parameter similar to the mutation operator in GA. Assuming $n$ chains are selected for cloning and $n_{c}$ clones are created per chain then $n X n_{c}$ chains are subjected to hypermutation operation according to the probability $p_{m}$. The probability $p_{m}$ is adaptive in nature and varies for certain number of iterations after being set to its original value.

In reselection process the $n$ best clones are selected from $n X n_{c}$ clones and placed in the current population. The replacement can be either greedy or non greedy. If we use a greedy method we replace the worst $n$ in the current population $P$ with the $n$ best clones. If we use a non greedy strategy then we replace the worst in particular intervals.

Fig.1. shows the block structure of the FLC optimizing process using evolutionary algorithms, in this case AIS. Similar to GA optimization of FLC, there are a total of 42 decision variables to be optimized. AIS searches for all the antecedent and consequent parameters in the d-dimensional space. lows:

The pseudo-code for optimization of FLC using CSA model of AIS is as fol-

1. Initialize the population with antibody chains with each chain being a solution of the FLC.

2. Evaluate the fitness of each of the antibody chains using Eq(5).

3. Select antibodies for cloning. Each antibody would have the same clone size, not privileging anyone for their affinity.

4. The antigen affinity(corresponding to fitness value) is used to determine the $\mathrm{p}_{\mathrm{m}}$ which is adaptive in nature.

5. For re-selection process, the best clones are selected to replace the current population and move on to the next generation.

6. Steps 2-5 are repeated till maximum number of generations is reached

\section{Results and Discussion}

In this section the FLC's tuned by GA and AIS are analyzed

\subsection{Cart Centering Problem}

For optimization of FLC for CCP using GA, a random population of chromosomes are created. The parameter values for GA are: Number of chromosomes per generation $=10$, Genetic operators: Cross over rate $60 \%$, Mutation rate $20 \%$, reproduction rate $20 \%$ and Number of generations $=1000$. The best RMSE value obtained was 0.1407 using GA. 
Similar to GA, an initial random population of antibodies are created for AIS, for optimization of FLC for CCP. The parameter values for AIS are: Number of antibodies $=22$, Number of antigens $=22$, Number of clones per candidate $=10$, $p_{m}=0.1$ and Number of generations $=1000$. The best RMSE value obtained was 0.0911 using AIS.

To compare the efficacy of AIS over GA a statistical test is done, in which each algorithm is run twenty times and the maximum, minimum, average and standard deviation of the RMSE values are noted, as shown in Table 2. We observe that AIS succeeds to a large extent in tuning a FLC over GA. It is also observed that AIS has a lower average RMSE value indicating that it is also consistent for the CCP. A low standard deviation obtained for AIS also indicates that there is very little divergence in the RMSE values obtained for the twenty trials. Fig2. shows the plot of cart position for the best design of GA and AIS. It can be observed that the FLC tuned by AIS brings the cart to zero position in under 0.5 seconds unlike GA which takes over 1.5 seconds. Fig. 3. Shows the plot of RMSE values for the best design of GA and AIS for CCP.

Once the FLC are tuned by GA and AIS, a study was made to determine their performance in the presence of noise. For this test we consider the best design of GA and AIS and add noise of power $10^{-6}$ and $10^{\circ}$. For FLC tuned by GA we obtain RMSE values of 0.1408 and 0.1910 for noise power of $10^{-6}$ and $10^{0}$ respectively. And for FLC tuned by AIS we obtain RMSE values of 0.0912 and 0.0946 for noise power of $10^{-6}$ and $10^{0}$ respectively. Thus indicating the superiority of AIS in obtaining a FLC which works well even in the presence of noise. The robustness of the FLC tuned by GA and AIS was tested for robustness by changing the mass of the cart .The RMSE values obtained for GA are $0.270607,0.3834$ and 0.579 and the RMSE values obtained for AIS are $0.105,0.2277$ and 0.3441 for mass of cart $5 \mathrm{~kg}, 10 \mathrm{~kg}$ and $20 \mathrm{~kg}$ respectively. This indicates that the FLC tuned by AIS is also robust. It was also necessary to know, how the FLC's performed in the presence of faulty rules. For this test three rules were chosen and purposely made faulty. Rule at location(row, coloumn) $(1,1),(2,2)$ and $(4,4)$ with respect to Table 1 are selected. The divergence errors(DE), which is the difference between the actual RMSE obtained with correct rule and RMSE obtained for faulty rule is noted. This is shown in Table 4 and Table 5 for GA and AIS respectively. It is noted that by changing rules on the edges $(1,1)$ will result in large DE while rules well placed within the rule base do not change the RMSE values. This can be attributed to the fact that rule $(1,1)$ do not have the luxury of having rules completely surrounding them and able to compensate for their mistakes.

The comparison of the performance of GA and AIS was also carried out using the Kruskal Wallis $(\mathrm{KW})$ rank test. In which the ranks for the respective algorithms are assigned. The lower the rank the better the algorithm. For CCP the ranks obtained for AIS and GA are 10.55 and 30.45 respectively and the Chi- sq is 28.976 and $p=0(p<0.05)$. Thus through $\mathrm{KW}$ test it is observed that AIS is better than GA for CCP.

A study was also made to understand the effect of the most important parameters of the algorithms. For GA, cross over rate and mutation rate play an important role and for AIS, it is the $p_{m}$ and the number of clones. For this study the random 
initial population was kept same for both GA and AIS so that comparison on a common platform is possible and the effect of individual parameters can be studied. For GA, by changing the cross over rates to $20 \%, 40 \%, 60 \%$ and $80 \%$ the RMSE values obtained is $0.240318,0.167296,0.126362$ and 0.139249 respectively. And by changing the number of genes undergoing mutation per chromosome to 1, 2, 3 and 4 RMSE values of $0.614012,0.305291,0.365119$ and 0.216281 were obtained. For AIS, the $p_{m}$ is changed to $0.1,0.15,0.2,0.25,0.3,0.35$ and 0.4 and the RMSE values obtained are $0.132462,0.148254,0.15662,0.173147,0.17584$, 0.225132 and 0.206485 respectively. The number of clones are changed to $3,5,7$ and 10 and the RMSE values obtained are 0.122387, 0.109719, 0.107266 and 0.101245 respectively. It is observed that the optimal crossover rate for GA is $60 \%$ and fitness value linearly becomes better with increase in mutation. It is also observed that for AIS the optimal $p_{m}$ is around 0.1 and fitness value linearly becomes better with increase in number of clones.

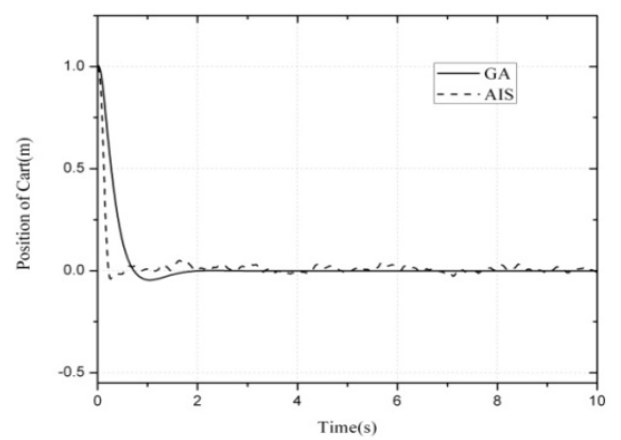

Fig. 2. Plot of Cart Position for the best design of GA and AIS.

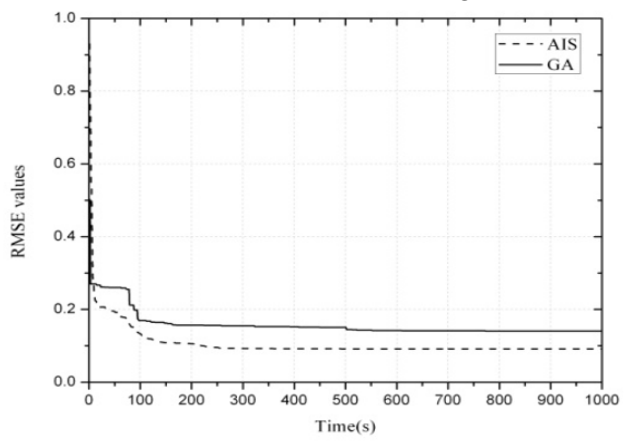

Fig. 3. Plot of RMSE values for the best design of GA and AIS for CCP

Table 2. Statistical Tests of fitness values for AIS and GA

\begin{tabular}{ccrrc}
\hline \hline Algorithms & Maximum & Minimum & Average & Standard Deviation \\
\hline AIS & 0.1461 & 0.0911 & 0.109 & 0.0162 \\
GA & 0.5116 & 0.1407 & 0.223 & 0.0836 \\
\hline \hline
\end{tabular}


Table 3. Presence of Faulty rules for FLC tuned by GA for CCP

\begin{tabular}{clcllll}
\hline \hline$(1,1)$ & $4-1$ & $4-2$ & $4-3$ & $4-5$ & $4-6$ & $4-7$ \\
$\mathrm{DE}$ & 0.7432 & 0.0045 & 0.1391 & 1.0043 & 0.3087 & 0.7432 \\
$(2,2)$ & $4-1$ & $4-2$ & $4-3$ & $4-5$ & $4-6$ & $4-7$ \\
$\mathrm{DE}$ & 0.0044 & 0 & 0.008 & 0.0053 & 0.002 & 0.0044 \\
$(4,4)$ & $4-1$ & $4-2$ & $4-3$ & $4-5$ & $4-6$ & $4-7$ \\
$\mathrm{DE}$ & 0 & 0 & 0 & 0 & 0 & 0 \\
\hline \hline
\end{tabular}

Where 1,2,3,4,5,6,7 are NB, NM, NS, Z, PS, PM and PB respectively.

Table 4. Presence of Faulty rules for FLC tuned by AIS for CCP

\begin{tabular}{ccccccc}
\hline \hline$(1,1)$ & $4-1$ & $4-2$ & $4-3$ & $4-5$ & $4-6$ & $4-7$ \\
DE & 0.0004 & 0.0896 & 0.0896 & 6.8238 & 0.0896 & 6.8782 \\
$(2,2)$ & $4-1$ & $4-2$ & $4-3$ & $4-5$ & $4-6$ & $4-7$ \\
DE & 0 & 0 & 0 & 0 & 0 & 0 \\
$(4,4)$ & $4-1$ & $4-2$ & $4-3$ & $4-5$ & $4-6$ & $4-7$ \\
DE & 0 & 0 & 0 & 0 & 0 & 0 \\
\hline \hline
\end{tabular}

\subsection{Inverted Pendulum}

For optimization of FLC for IP using GA and AIS the parameters are the same as chosen for CCP. The total number of generations chosen is 100 . To compare the efficacy of AIS over GA for the IP a statistical test is done similar to CCP as shown in Table 5. We observe that here too AIS succeeds to a large extent in tuning an optimal FLC over GA as the maximum, minimum and average RMSE for AIS being lower than GA. Further the Standard deviation of AIS is also lesser than GA. Fig.4. shows the plot of pendulum position for the best design of GA and AIS. It can be observed that the FLC tuned by AIS brings the pendulum to zero position in under 0.5 seconds, while GA stabilizes the pendulum to a value slightly less than 0 in over 0.75 seconds.

A study to determine the performance of FLC tuned by GA and AIS in the presence of noise is made. For this test we consider the best design of GA and AIS and add noise of power $10^{-6}$ and $10^{-9}$. For FLC tuned by GA we obtain RMSE values of 0.019677 and 0.01737 for noise power of $10^{-6}$ and $10^{-9}$ respectively. And for FLC tuned by AIS we obtain RMSE values of 0.018067 and 0.016725 for noise power of $10^{-6}$ and $10^{-9}$ respectively. Thus indicating the superiority of AIS in obtaining a FLC which works well even in the presence of noise. The robustness of the FLC tuned by GA and AIS was tested for robustness by changing the mass of the pendulum and length of the pendulum to three times its initial value. The RMSE values obtained for GA are 0.020708 and 0.0207 and the RMSE values obtained for AIS are 0.015168 and 0.0189 for mass of pendulum $0.3 \mathrm{~kg}$ and length of pendulum $1.5 \mathrm{~m}$ respectively. This indicates that the FLC tuned by AIS is also robust. A test of the FLC's performance in the presence of faulty rules was also conducted. For this test the same three rules were chosen as in the case of CCP. This is shown in Table 6 and 7 for GA and AIS respectively. It is noted that by changing rules on the edges $(1,1)$ will result in large DE while rules well placed within 
the rule base do not change the RMSE values. For IP we obtained the ranks for AIS and GA to be 7.30 and 13.70 respectively and the chi-sq is 5.8157, $\mathrm{p}=0.016(\mathrm{p}<0.05)$. Thus for IP, AIS is better than GA due to its lower rank.

A study was also made to understand the effect of the most important parameters of the algorithms similar to the CCP. For GA, by changing the cross over rates to $20 \%, 40 \%, 60 \%$ and $80 \%$ the RMSE values obtained is $0.01735,0.022517$, 0.023607 and 0.026612 respectively. And by changing the number of genes undergoing mutation per chromosome to $1,2,3$ and 4 RMSE values of 0.017977 , $0.021193,0.022882$ and 0.019445 were obtained. For AIS, the $p_{m}$ is changed to $0.1,0.15,0.2,0.25,0.3,0.35$ and 0.4 and the RMSE values obtained are 0.020537 , $0.018752,0.017945,0.021381,0.018301,0.019726$ and 0.023414 respectively. The numbers of clones are changed to 3, 5, 7 and 10 and the RMSE values obtained are $0.01843,0.014206,0.014151$ and 0.01335 respectively. It is observed that the optimal crossover rate for GA is $20 \%$ and optimal mutations per chromosome is 1 . It is also observed that for AIS the optimal $p_{m}$ is around 0.2 and fitness value linearly becomes better with increase in number of clones as was observed for CCP. With these tests, it is clear that the optimal parameters of either GA or AIS are problem dependent and no definitive pattern can be obtained, except for the number of clones in AIS. This is logical as increase in number if clones increases the local search around an optimal point which could result in a better solution.

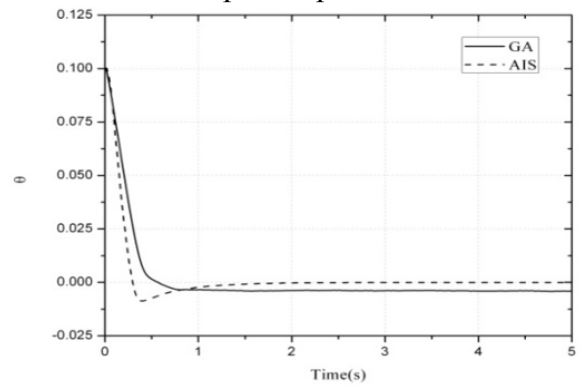

Fig.4 Trajectory Plot of Cart Position for the best design of GA and AIS

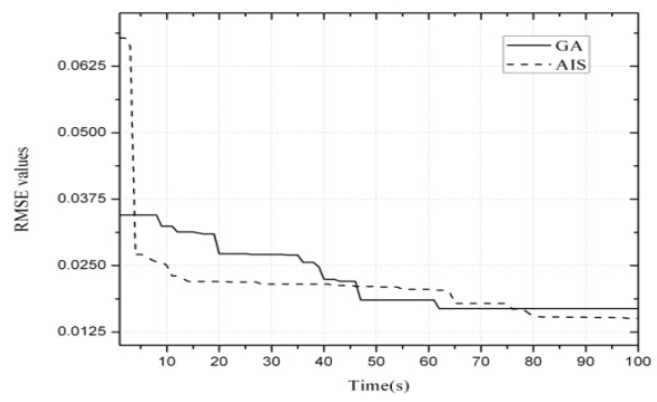

Fig.5 Plot of RMSE values for the best design of GA and AIS for IP 
Table 5. Statistical Tests of fitness values for AIS and GA

\begin{tabular}{ccccc}
\hline \hline Algorithms & Maximum & Minimum & Average & Standard Deviation \\
\hline AIS & 0.0151 & 0.0256 & 0.0196 & 0.0027 \\
GA & 0.0182 & 0.02510 & 0.0229 & 0.0018 \\
\hline \hline
\end{tabular}

Table 6. Presence of Faulty rules GA for IP

\begin{tabular}{clllclc}
\hline \hline$(1,1)$ & $4-1$ & $4-2$ & $4-3$ & $4-5$ & $4-6$ & $4-7$ \\
$\mathrm{DE}$ & 0.1067 & 0.8994 & 0.0162 & 0.0012 & 0.0076 & 0 \\
$(2,2)$ & $4-1$ & $4-2$ & $4-3$ & $4-5$ & $4-6$ & $4-7$ \\
$\mathrm{DE}$ & 0.0061 & 0.007 & 0.0002 & 0 & 0.0094 & 0.0005 \\
$(4,4)$ & $4-1$ & $4-2$ & $4-3$ & $4-5$ & $4-6$ & $4-7$ \\
$\mathrm{DE}$ & 0 & 0 & 0 & 0 & 0 & 0 \\
\hline \hline
\end{tabular}

Table 7. Presence of Faulty rules AIS for IP

\begin{tabular}{clrrrrl}
\hline \hline$(1,1)$ & $4-1$ & $4-2$ & $4-3$ & $4-5$ & $4-6$ & $4-7$ \\
$\mathrm{DE}$ & 0.4023 & 0.4015 & 0.0024 & 0 & 0.2571 & 0.0011 \\
$(2,2)$ & $4-1$ & $4-2$ & $4-3$ & $4-5$ & $4-6$ & $4-7$ \\
$\mathrm{DE}$ & 0.0002 & 0 & 0 & 0 & 0 & 0.0001 \\
$(4,4)$ & $4-1$ & $4-2$ & $4-3$ & $4-5$ & $4-6$ & $4-7$ \\
$\mathrm{DE}$ & 0 & 0 & 0 & 0 & 0 & 0 \\
\hline \hline
\end{tabular}

\section{Conclusions}

In this paper, the Clonal selection Algorithm(CSA) a model of the Artificial Immune System(AIS) paradigm for tuning the antecedent and consequent parameters of the membership functions of a Mamdani type fuzzy Logic controller is examined. Two problems were considered, firstly the cart centering problem and secondly the classic benchmark in control, the inverted pendulum problem. The FLC tuned by AIS was compared with a FLC tuned by GA for both the problems considered. The FLC once tuned by the two algorithms were then checked for robustness by adding white noise of varying power densities to the system. Robustness was also checked by varying the mass of the cart and the mass and the length of the pendulum. The FLC's were also tested in the presence of faulty rules. It was observed that the FLC tuned by AIS outperforms the FLC tuned by GA for all cases. The Kruskall Wallis test performed, has also proved that AIS is a better algorithm for optimization compared to GA. An insight into the effect of individual parameters of GA and AIS was also conducted. 


\section{References}

[1] Zafer Bingul, Oguzhan Karahan: A Fuzzy Logic Controller tuned with PSO for 2 DOF robot trajectory control. Eng. Appl. Artif. Intel. 38 (1), 1017-1031(2011)

[2] Lal Bahadur Prasad, Hari Om Gupta, Barjeev Tyagi: Intelligent control of nonlinear inverted pendulum dynamical system with disturbance input using fuzzy logic systems. International Conference on Recent Advancements in Electrical, Electronics and Control Engineering (2011)

[3] Arup Kumar Nandi, J. Paulo Davim: A study of drilling performances with minimum quantity of lubricant using fuzzy logic rules. Mechatronics.19,218-232 (2009)

[4] Olga Diamante, Giovanna Fargione, Antonino Risitano, Domenico Tringali: A soft computing approach to fuzzy sky-hook control of semiactive suspension. IEEE T. Contr. Syst. T. 11 (6), (2003)

[5] Un-Chul Moon; Kwang Y; Lee: Hybrid algorithm with fuzzy system and conventional PI control for the temperature control of TV glass furnace. IEEE T. Contr. Syst. T. 11(4), (2003)

[6] Timothy J. Ross: Fuzzy logic with engineering applications. John Wiley (2004)

[7] Belarbi, K., Titel F., Bourebi W., Benmahammed, K.: Design of mamdani fuzzy logic controllers with rule base minimisation using genetic algorithm, Eng. Appl. Artif. Intel. 18(7),875-880 (2005)

[8] Lam, H.K., Leung, F.H.F. Tam, P.K.S.: Design and stability analysis of fuzzy model based nonlinear controller for nonlinear systems using genetic algorithm. Proceedings of the IEEE International Conference on Fuzzy Systems 1,232 - 237 (2002)

[9] Leung, F.H.F. , Lam, H.K. , Ling, S.H., Tam, P.K.S. :Optimal and stable fuzzy controllers for nonlinear systems based on an improved genetic algorithm. IEEE T. Ind Electron. 51( 1 ), 182 - 192(2004)

[10] Emad Elbeltagi, Tarek Hegazy, Donald Grierson,: Comparison among five evolutionary based optimization algorithms. Adv. Eng. Info. 19,43-53(2005)

[11] Omkar, S.N., Rahul Khandelwal, Santosh Yathindra,Narayana Naik,G., Gopalakrishnan :Artificial immune system for multi-objective design optimization of composite structures. Eng. Appl. Artif. Intel. 21 (8), 1416-1429(2008)

[12] Pengfei Liu, Ping Xu, Jinyang Zheng:Artificial Immune System for optimal design of composite hydrogen vessel. Comput. Mater. Sci. 47 (1), 261-267(2009)

[13] Zhuhong Zhang, Shuqu Qian: Artificial immune system in dynamic environments solving time-varying non linear constrained multi-objective problems. Soft Comput. $15,1333-1349(2011)$

[14] Taur,J.S, Tzuen Wuu Hsieh, Tsai,C.L.: design of a Fuzzy Controller with Fuzzy Swing Up and Parallel distributed Pole Assignment Schemes for an Inverted Pendulum and Cart System. IEEE T. Contr. Syst. T. 16 (6), 1277-1288(2008)

[15] El-Hawwary, M.I: Adaptive Fuzzy Control of the Inverted Pendulum Problem. IEEE T. Contr. Syst. T. 14(6), 1135-1144(2006)

[16] Henry Lau, Y.K., Vicky Wong, W.K.: An immunity approach to strategic behavioral control. Eng. Appl. Artif. Intel.20 (3), 289-306(2007) 